\title{
Continuous spinal an- aesthesia for abdominal surgery in a patient receiving amiodarone
}

\begin{abstract}
The perioperative management of a 57-yr-old patient receiving chronic amiodarone therapy with a continuous spinal anaesthetic for a low anterior resection of the colon is discussed. The most appropriate anaesthetic technique for patients receiving chronic amiodarone therapy remains controversial, but the avoidance of general anaesthesia may be beneficial because of the risk of postoperative pulmonary failure. In this patient continuous spinal anaesthesia was slowly titrated to the desired level, coincident haemodynamic alterations were easily treated as they developed, and high serum local anaesthetic concentrations which occur with other regional andesthetic techniques were avoided.
\end{abstract}

La conduite périopératoire d'une anesthésie rachidienne continue lors d'une résection antérieure basse du côlon chez un patient âgé de 57 ans recevant une thérapie chronique $\dot{a}$ l'amiodarone est décrite. La technique anesthésique la plus appropriée pour les patients. sous thérapie chronique d̀ l'amiodarone demeure controversée, mais le fait d'éviter l'anesthésie générale pourrait être bénéfique à cause du risque d'insuffisance pulmonaire postopératoire. Chez ce patient, l'anesthésie rachidienne continue est lentement titrée au niveau désiré et less altérations hémodynamiques furent facilement traitées au fur et à mesure qu'ils apparaissent et l'augmentation des concentrations sériques et des anesthésiques locaux qui surviennent avec d'autres techniques d'anesthésie régionale furent évités.

\section{Key words}

ANAESTHETIC TECHNIQUES: spinal-continuous; HEART: antiarrhythmics; amiodarone;

INTERACTIONS (DRUG): amiodarone, anaesthetic agents.

From the Department of Anesthesiology, Virginia Mason Medical Center, Seattle, Washington.

Address correspondence to: Dr. D. J. Kopacz, Department of Anesthesiology, Virginia Mason Clinic, P.O. Box 900 , Seattle, WA 98111.

Accepted for publication 27 th November 1990.
Amiodarone is a class III antiarrhythmic agent whose key actions include prolongation of the action potential and effective refractory period of the heart. ' Although it is the most effective oral agent available for treating patients with ventricular tachycardia, toxicity has limited its initial use to patients whose arrythmias are not effectively treated by other agents.

Amiodarone-induced pulmonary toxicity has been reported to occur in $5-28 \%$ of patients using this drug chronically. ${ }^{2}$ The clinical course is usually characterized by a slow subacute onset which reverses on reduction of drug dosage, termination of therapy, or corticosteroid administration.

Fulminant life-threatening or fatal adult respiratory distress syndrome has been reported after general anaesthesia in patients receiving amiodarone, even in the absence of apparent preoperative pulmonary damage. ${ }^{3,4}$ As the patient population at risk of developing adverse reactions to amiodarone is not defined, the perioperative management of patients taking this agent remains controversial. ${ }^{5}$ Avoidance of general anaesthesia may be advantageous. This report describes the clinical course of a patient receiving chronic amiodarone therapy in which continous spinal anaesthesia was utilized for major abdominal surgery.

\section{Case report}

A 57-yr-old male receiving amiodarone was scheduled for anterior resection of a partially obstructing adenocarcinoma of the colon. He had a history of congestive cardiomyopathy since 1983 (gated pool LVEF $=34 \%, \mathrm{RVEF}=$ $52 \%$ ). Three years before admission, he had an episode of ventricular fibrillation-induced cardiac arrest. The arrhythmias were refractory to multiple conventional antiarrhythmic regimens. Amiodarone (alternating 200 and 400 $\mathrm{mg} p o$ daily) was eventually instituted to suppress the arrythmias. He had no known primary lung disease, was a non-smoker, and his remaining medical history was noncontributory. He was taking no other medications.

Preoperative physical examination revealed a $175 \mathrm{~cm}$, $72 \mathrm{~kg}$ man with blood pressure (BP) ranging from 120/60 to $135 / 80 \mathrm{mmHg}$ and heart rate (HR) from $65-85$ 
TABLE Haemodynamic changes during abdominal surgery with continuous spinal anacsthesia (bupivacaine) in a patient receiving amiodarone

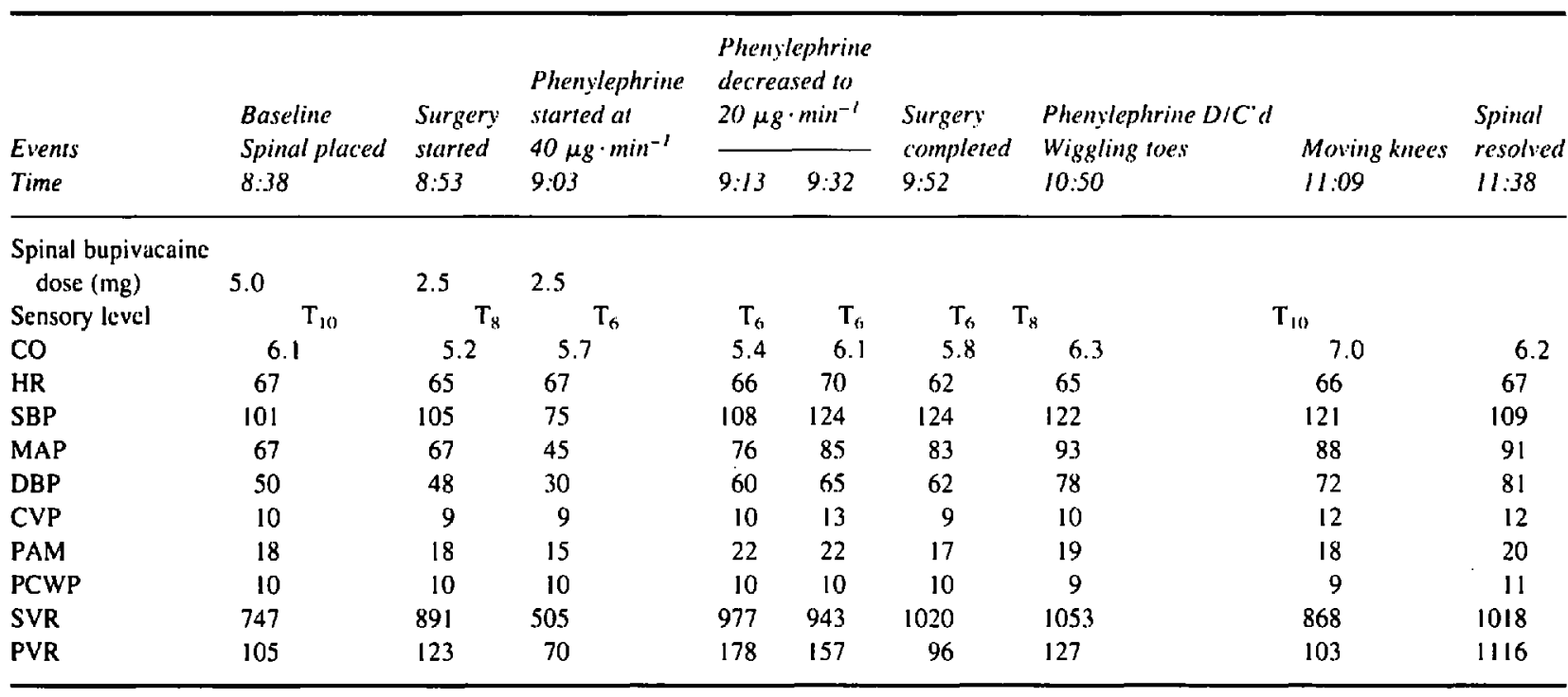

beats $\cdot \min ^{-1}$. Apart from a laterally displaced apical impulse his physical examination was unremarkable.

Electrocardiogram showed sinus rhythm with an occasional ventricular ectopic beat and a first degree atrioventricular block at a rate of 68 beats $\cdot \mathrm{min}^{-1}$. Chest roentgenogram revealed a slightly enlarged cardiac shadow and was free of interstitial infiltrates. Pulmonary function studies showed $\mathrm{FEV}_{1}=3.49$ 1. (103\% predicted), and $\mathrm{FVC}=4.601$. (75\% predicted). Other routine laboratory tests were normal.

Amiodarone was continued until the morning of surgery $(200 \mathrm{mg})$. No premedication was given before the patient's arrival at the operating suite. Following placement of non-invasive monitors and delivery of $2 \mathrm{~L} \cdot \mathrm{min}^{-1}$ of supplemental oxygen by nasal cannula, mild sedation was established with $2 \mathrm{mg}$ iv midazolam. With ventricular pacing capability readily available, invasive monitoring was established with a radial arterial catheter and a thermodilution pulmonary artery (PA) catheter placed via the right internal jugular vein.

A 20 ga catheter was easily placed $4 \mathrm{~cm}$ into the subarachnoid space via an $18 \mathrm{~g}$. Tuohy needle with its needle bevel oriented parallel to the long axis of the dural sac. After obtaining baseline haemodynamic measurements, spinal anaesthesia was induced with $5.0 \mathrm{mg}$ hyperbaric bupivacaine $(0.75 \%$ in $8.25 \%$ dextrose without epinephrine) and slowly titrated over a 15 -min period to a sensory level of $\mathrm{T}_{6}$ using incremental doses of $2.5 \mathrm{mg}$ (10 mg total). Intermittent haemodynamic measurements taken during this period demonstrated a gradual decrease in mean arterial pressure (MAP) after institution of spinal anaesthesia, and a corresponding further decrease in calculated systemic vascular resistance (SVR) below its initially low baseline level (Table). Titration of a phenylephrine infusion returned MAP and SVR to normal. Minimal changes in cardiac output occurred during these interventions.

The surgical procedure was completed in one hour with an estimated $150 \mathrm{ml}$ blood loss replaced with $1600 \mathrm{ml}$ iv normal saline. The anaesthetic level remained at $T_{6}$ throughout surgery. The phenylephrine infusion was tapered and discontinued in the recovery room as the segmental analgesic level regressed. The PA catheter was removed prior to discharge from the recovery room.

On the second postoperative day, the patient developed mild symptoms of a typical postdural puncture headache which resolved spontaneously that evening without treatment.

On the third postoperative day, the patient's wound below the umbilicus dehisced and he was returned to the operating room for wound closure. With knowledge of the patient's response to his previous anaesthetic, it was decided not to replace the PA catheter, and to administer a single injection spinal anaesthetic. Intrathecal injection of $10 \mathrm{mg}$ plain bupivacaine produced a sensory block to $T_{8}$ and wound closure was completed uneventfully. Four incremental injections of $50 \mu \mathrm{g}$ phenylephrine were given during surgery to maintain haemodynamic stability. His subsequent recovery was uneventful and he was discharged ten days after his original surgery in stable health.

\section{Discussion}

Amiodarone-induced pulmonary toxicity is well documented. Rapid acceleration of this syndrome with the development of acute fulminant respiratory insufficiency 
early after general anaesthesia has recently been described. Tuzcu et al. reported a higher incidence of postoperative pulmonary failure and longer duration of tracheal intubation and intensive care stay in postoperative cardiac surgery patients who were treated chronically with amiodarone. ${ }^{6}$ Kay et al. reported four patients who developed fatal respiratory failure after noncardiac surgery. ${ }^{3}$ All did well immediately after surgery, but rapidly developed signs and symptoms of pulmonary insufficiency 18 to 48 hr after tracheal extubation.

The long elimination half-life of amiodarone (52 d) makes the preoperative discontinuation of the drug impractical for many surgical procedures. ${ }^{2}$ Discontinuation of the drug may also result in recurrence of the life-threatening arrhythmias. Furthermore, fulminant pulmonary dysfunction may occur after general anaesthesia, even after lengthy discontinuation of amiodarone. ${ }^{4}$ The use of a regional anaesthetic technique and avoidance of general anaesthesia could therefore be beneficial to these patients.

Severe pharmacodynamic interactions have been described between amiodarone and type I antiarrhythmic agents, such as lidocaine. Additive prolongation of the QT interval, development of torsades de pointes, and a $30-50 \%$ increase in serum antiarrythmic concentration have been reported. ${ }^{7}$ Several cases of intraoperative sinus arrest, severe atropine-resistant bradycardia, and conplete heart block have now been reported in patients receiving amiodarone during anaesthesia. ${ }^{8.9}$ Nodal rhythm and/or complete heart block developed intraoperatively in ten of 15 patients reported by Liberman. ${ }^{10}$ In the presence of acute amiodarone-induced complete heart block, local anaesthetics with type I antiarrhythmic properties could suppress the escape rhythm from lower pacemakers and result in cardiac arrest.

Spinal anaesthesia is a particularly attractive regional anaesthetic technique in the patient receiving amiodarone because it avoids exposure to high systemic levels of local anaesthetic. Although serum levels are only slightly higher after the epidural injection of a low dose (100 mg) of lidocaine than after an equivalent dose is given into the subarachnoid space, ${ }^{11}$ much larger volumes of local anaesthetic are generally necessary for epidural anaesthesia. This eventually yields serum levels, for either lidocaine or bupivacaine, that are four- to ten-fold higher after epidural than after spinal anaesthesia. ${ }^{12.13}$ There is no evidence that serum concentrations attained after spinal anaesthesia are cardioactive.

The decrease in mean arterial pressure and calculated SVR in this patient were attributed to sympathetic blockade superimposed upon the baseline amiodaroneinduced noncompetitive $\alpha$ adrenergic block. A phenylephrine infusion was necessary to return SVR to baseline and to maintain the pulmonary capillary wedge pressure between $9-10 \mathrm{~cm} \mathrm{H}_{2} \mathrm{O}$. One might expect cardiac output to increase in the presence of constant left-sided filling pressures after the spinal-induced afterload reduction. Amiodarone-induced reduction in cardiac systolic reserve possibly prevented an increase in cardiac output in this patient. Laboratory investigation in dogs suggests that epinephrine may be the most effective agent to reverse the myocardial depressive effect of amiodarone, but this remains unproven in the clinical setting. ${ }^{14}$

Amiodarone possesses a multitude of electrophysiological actions and produces a noncompetitive adrenergic blockade, greatly reducing the effectiveness of sympathetic agents at both $\alpha$ and $\beta$ receptors. ${ }^{15}$ Testing the responsiveness of $\alpha$ adrenergic agents before administering anaesthesia to patients on amiodarone has been suggested. ${ }^{16}$ Establishing the responsiveness to $\beta$ adrenergic agents may also be beneficial, as has been suggested for patients on chronic $\beta$ blocker therapy. ${ }^{17}$ As amiodarone may also directly affect intracardiac conduction, ventricular pacing must also be necessary.

The recent development of smaller gauge spinal needles and catheters suggests that a rebirth of the use of continuous spinal anaesthesia may occur. Hurley and Lambert recently reviewed the advantages and disadvantages of continuous spinal anaesthesia, using their new 32-gauge microcatheter. ${ }^{18}$ In patients with severe cardiovascular disease, a subarachnoid catheter is especially advantageous compared with a single-dose spinal technique. Small incremental doses of drug can be slowly titrated to a desired anaesthetic level and balanced against the coincident haemodynamic changes, for which vasoactive drugs can be instituted if necessary.

In conclusion, a case is presented in which the use of continuous spinal anaesthesia provided a safe, controllable alternative to general anaesthesia in a patient receiving amiodarone therapy. The advantages of using continuous spinal anaesthesia in the subgroup of patients with a history of severe cardiovascular problems having surgical procedures of the abdomen or lower extremities are discussed.

\section{References}

1 Marcus FI, Fontaine GH, Frank R, Grosgogeat $\gamma$. Clinical pharmacology and therapeutic applications of the antiarrhythmic agent, amiodarone. Am Heart J 1981; 101: 480-93.

2 Mason JW. Amiodarone. N Engl J Med 1987; 316 455-66.

3 Kay GN, Epstein AE, Kirklin JK, Diethelm AG, Graybar $G$, Plumb VJ. Fatal postoperative amiodarone pulmonary toxicity. Am J Cardiol 1988; 62: 490-2. 
4 Nalos PC, Kass RM, Gang ES, Fishbein MC, Manjel WJ, Peter $T$. Life-threatening postoperative pulmonary complications in patients with previous amiodarone pulmonary toxicity undergoing cardiothoracic operations. J Thorac Cardiovasc Surg 1987; 93: 904-12.

5 Teasdale $S$. Amiodarone and anacsthesia. Can J Anacsth 1990; 37: 151-4.

6 Tuzcu EM, Maloney JD, Sangai BH et al. Cardiopulmonary effects of chronic amiodarone "ierapy in the carly postoperative course of cardiac s' .gery patients. Cleve Clin J Med 1987; 54: 491-5.

7 Marcus FI. Drug interactions with amiodarone. Am Heart J 1983; 106: 924-9.

8 Gallagher JD, Lieberman RW, Meranze J, Spielman SR, Elison N. Amiodaronc-induced complications during coronary artery surgery. Anesthesiology 1981; 55: 186-8.

9 Navalgund AA, Alifimoff $J K$, Jakemec AJ, Bleyaert $A L$. Amiodarone-induced sinus arrest successfully treated with ephedrine and isoproterenol. Ancsth Analg 1986; 65: 414-6.

10 Liberman BA, Teasdale SJ. Anaesthesia and amiodaronc. Can Anaesth Soc J 1985; 32: 629-38.

11 Giasi RM, D'Agostino E. Covino BG. Absorption of lidocaine following subarachnoid and epidural administration. Anesth Analg 1979; 58: 360-3.

12 Burm AGL, van Kleef JW, Gladines MPRR, van Duinen $M$, Spierdijk $J$. Spinal anesthesia with hyperbaric lidocaine and bupivacainc: effects of epinephrine on the plasma concentration profiles. Anesth Analg 1987; 66: 1104-8.

13 Burm AGL, van Kleef JW. Gladines MPRR, Olthof $G$, Spierdijk J. Epidural anesthesia with lidocaine and bupivacaine: effects of epinephrine on the plasma concentration profiles. Anesth Analg 1986; 65: 1281-4.

14 Spotnitz WD, Nolan SP, Kaiser DL et al. The reversal of amiodarone-induced perioperative reduction in cardiac systolic reserve in dogs. J Am Coll Cardiol 1988; 12: 757-64.

15 Charlier $R$. Cardiac actions in the dog of a new antagonist of adrenergic cxcitation which does not produce competitive blockade of adrenoceptors. Br J Pharmacol 1970; 39: 674-88.

16 Koblin DD, Romanoff ME, Martin DE et al. Anesthetic management of the parturient receiving amiodarone. Anesthesiology 1987; 66: 551-3.

17 Dagnino J, Prys-Roberts $C$. Assessment of $\beta$ adrenoceptor blockade during anesthesia in humans: use of isoproterenol dose-response curves. Anesth Analg 1985; 64: 305-11.

18 Hurley $R J$, Lambert $D H$. Continuous spinal anesthesia with a microcatheter technique: preliminary experience. Anesth Analg 1990; 70: 97-102. 